\title{
ARITHMETIC PROPERTIES OF 1-SHELL TOTALLY SYMMETRIC PLANE PARTITIONS
}

\author{
MICHAEL D. HIRSCHHORN and JAMES A. SELLERS ${ }^{\bowtie}$
}

(Received 8 July 2013; accepted 31 July 2013; first published online 27 September 2013)

\begin{abstract}
Blecher ['Geometry for totally symmetric plane partitions (TSPPs) with self-conjugate main diagonal', Util. Math. 88 (2012), 223-235] defined the combinatorial objects known as 1-shell totally symmetric plane partitions of weight $n$. He also proved that the generating function for $f(n)$, the number of 1-shell totally symmetric plane partitions of weight $n$, is given by

$$
\sum_{n \geq 0} f(n) q^{n}=1+\sum_{n \geq 1} q^{3 n-2} \prod_{i=0}^{n-2}\left(1+q^{6 i+3}\right)
$$

In this brief note, we prove a number of arithmetic properties satisfied by $f(n)$ using elementary generating function manipulations and well-known results of Ramanujan and Watson.

2010 Mathematics subject classification: primary 05A17; secondary 11P83.

Keywords and phrases: partition, totally symmetric plane partition, TSPP, generating function, congruence.
\end{abstract}

\section{Introduction}

In 2012, Blecher [4] considered a special class of totally symmetric plane partitions (TSPPs) which he called 1-shell TSPPs. (For more information about TSPPs in general, the reader may refer to $[3,7]$.) As defined by Blecher, a 1-shell TSPP has a self-conjugate first row/column (as an ordinary partition) and all other entries are 1. For example, the following are 1-shell TSPPs:

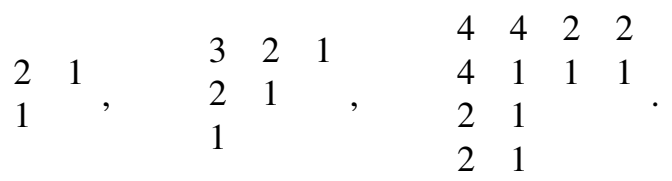

J. A. Sellers thanks the John Knopfmacher Centre for Applicable Analysis and Number Theory for their generous support which allowed him to visit the University of Witwatersrand in May 2013. It was at this time that this work was initiated.

(c) 2013 Australian Mathematical Publishing Association Inc. 0004-9727/2013 \$16.00 
In this paper, we let $f(n)$ be the number of 1-shell TSPPs with weight $n$ (so that the parts of the TSPP sum to $n$ ). In [4], Blecher proved that

$$
F(q):=\sum_{n \geq 0} f(n) q^{n}=1+\sum_{n \geq 1} q^{3 n-2} \prod_{i=0}^{n-2}\left(1+q^{6 i+3}\right) .
$$

Our goal in this brief work is to prove a number of arithmetic properties satisfied by $f(n)$. To prove these results, we will use (1.1) along with a number of wellknown identities of Ramanujan and Watson (and some elementary generating function manipulations).

\section{Arithmetic properties}

We begin with an extremely straightforward result which follows from (1.1).

Theorem 2.1. For all $n \geq 1$, if $n \equiv 0,2(\bmod 3)$, then $f(n)=0$.

Proof. Note that when the right-hand side of (1.1) is written as a power series in $q$, the only powers of $q$ which appear are of the form $q^{3 j+1}$ for some $j \geq 0$. This immediately proves the result.

Next, we transition to a parity result for $f(n)$ which provides a nice characterisation based on the prime factorisation of $n$.

Theorem 2.2. For all $n \geq 1$,

$$
f(n) \equiv \begin{cases}1(\bmod 2) & \text { if } 3 \nmid n \text { and } n=k^{2} \text { for some integer } k, \\ 0(\bmod 2) & \text { otherwise. }\end{cases}
$$

Proof. Our proof begins with an identity of Ramanujan found in the work of Andrews and Berndt [2, Entry 9.5.2, page 238]:

$$
\sum_{n \geq 0} q^{n} \prod_{i=0}^{n-1}\left(1-q^{2 i+1}\right)=\sum_{n \geq 0}(-1)^{n} q^{3 n^{2}+2 n}\left(1+q^{2 n+1}\right) .
$$

Replacing $q$ by $q^{3}$ throughout (2.1) gives

$$
\sum_{n \geq 0} q^{3 n} \prod_{i=0}^{n-1}\left(1-q^{6 i+3}\right)=\sum_{n \geq 0}(-1)^{n} q^{9 n^{2}+6 n}\left(1+q^{6 n+3}\right) .
$$

We then multiply both sides of (2.2) by $q$ and add 1 to obtain

$$
\begin{aligned}
1+\sum_{n \geq 0} q^{3 n+1} \prod_{i=0}^{n-1}\left(1-q^{6 i+3}\right) & =1+\sum_{n \geq 0}(-1)^{n} q^{9 n^{2}+6 n+1}\left(1+q^{6 n+3}\right) \\
& =1+\sum_{n \geq 0}(-1)^{n}\left(q^{(3 n+1)^{2}}+q^{(3 n+2)^{2}}\right)
\end{aligned}
$$


which is equivalent to

$$
1+\sum_{n \geq 1} q^{3 n-2} \prod_{i=0}^{n-2}\left(1-q^{6 i+3}\right)=1+\sum_{n \geq 0}(-1)^{n}\left(q^{(3 n+1)^{2}}+q^{(3 n+2)^{2}}\right) .
$$

Thanks to (1.1) and (2.3) we have, modulo 2,

$$
\begin{aligned}
\sum_{n \geq 0} f(n) q^{n} & \equiv 1+\sum_{n \geq 1} q^{3 n-2} \prod_{i=0}^{n-2}\left(1-q^{6 i+3}\right) \\
& \equiv 1+\sum_{n \geq 0}\left(q^{(3 n+1)^{2}}+q^{(3 n+2)^{2}}\right)
\end{aligned}
$$

The result follows.

It is clear that Theorem 2.2 can be used to write down infinitely many Ramanujanlike congruences modulo 2. For example, we have the following corollaries.

COROLlaRy 2.3. Let $p \geq 5$ be a prime and let $r$ be any nonquadratic residue modulo $p$. Then, for all $n \geq 0$,

$$
f(p n+r) \equiv 0(\bmod 2) .
$$

Corollary 2.4. For all $m \geq 1, k=2,3,5,6$ and 7 , and all $n \geq 0$,

$$
f\left(m^{2}(8 n+k)\right) \equiv 0(\bmod 2) .
$$

Proof. This result follows because all squares are congruent to 0,1 , or 4 modulo 8 .

We now transition to consider an unexpected congruence satisfied by $f(n)$ modulo 5 .

Theorem 2.5. For all $n \geq 0, f(10 n+5) \equiv 0(\bmod 5)$.

Proof. First, thanks to Theorem 2.1, we know that $f(10(3 n)+5)=f(30 n+5)=0$ because $30 n+5 \equiv 2(\bmod 3)$ and $f(10(3 n+1)+5)=f(30 n+15)=0$ because $30 n+$ $15 \equiv 0(\bmod 3)$. Thus, the only thing needed to prove Theorem 2.5 is to confirm that $f(10(3 n+2)+5)=f(30 n+25) \equiv 0(\bmod 5)$ for all $n \geq 0$.

To begin our proof, we consider the third-order mock theta function

$$
v(q):=\sum_{n \geq 0} \frac{q^{n(n+1)}}{(1+q)\left(1+q^{3}\right) \cdots\left(1+q^{2 n+1}\right)}
$$

(as defined by Watson) which appears in Fine [5, Equation (26.82), page 61].

If we define the function $h(n)$ by

$$
\sum_{n \geq 1} h(n) q^{n}:=\sum_{n \geq 1} q^{n} \prod_{i=0}^{n-2}\left(1+q^{2 i+1}\right)
$$


then, thanks to Fine [5, Equation (26.85), page 61],

$$
\sum_{n \geq 1} h(n) q^{n}=q v(-q) .
$$

Fine [5, Equation (26.88), page 62] also notes that Watson proved

$$
v(-q)-q \omega\left(q^{2}\right)=\prod_{n \geq 1}\left(1+q^{2 n}\right)^{3}\left(1-q^{2 n}\right),
$$

where $\omega(q)$ is another of the third-order mock theta functions noted by Fine [5, Equation (26.81), page 61]. (The actual definition of $\omega(q)$ is not necessary for our purposes here.) Using (2.6),

$$
q v(-q)=q^{2} \omega\left(q^{2}\right)+q \prod_{n \geq 1}\left(1+q^{2 n}\right)^{3}\left(1-q^{2 n}\right) .
$$

From (2.5) and (2.7),

$$
\sum_{n \geq 1} h(n) q^{n}=q^{2} \omega\left(q^{2}\right)+q \prod_{n \geq 1}\left(1+q^{2 n}\right)^{3}\left(1-q^{2 n}\right) .
$$

If we extract the odd powers of $q$,

$$
\sum_{n \geq 0} h(2 n+1) q^{2 n+1}=q \prod_{n \geq 1}\left(1+q^{2 n}\right)^{3}\left(1-q^{2 n}\right)
$$

or

$$
\sum_{n \geq 0} h(2 n+1) q^{n}=\prod_{n \geq 1}\left(1+q^{n}\right)^{3}\left(1-q^{n}\right) .
$$

We now manipulate the right-hand side of (2.8) as follows:

$$
\begin{aligned}
\prod_{n \geq 1}\left(1+q^{n}\right)^{3}\left(1-q^{n}\right) & =\prod_{n \geq 1} \frac{\left(1+q^{n}\right)^{3}\left(1-q^{n}\right)\left(1-q^{n}\right)^{3}}{\left(1-q^{n}\right)^{3}} \\
& =\prod_{n \geq 1} \frac{\left(1-q^{2 n}\right)^{3}\left(1-q^{n}\right)}{\left(1-q^{n}\right)^{3}} \\
& =\prod_{n \geq 1} \frac{\left(1-q^{2 n}\right)^{3}\left(1-q^{n}\right)^{3}}{\left(1-q^{n}\right)^{5}} \\
& \equiv \prod_{n \geq 1} \frac{\left(1-q^{2 n}\right)^{3}\left(1-q^{n}\right)^{3}}{\left(1-q^{5 n}\right)}(\bmod 5) .
\end{aligned}
$$

We now wish to consider the power series for

$$
\prod_{n \geq 1}\left(1-q^{2 n}\right)^{3}\left(1-q^{n}\right)^{3}
$$


modulo 5. We have the well-known result of Jacobi [1, page 176] which states that

$$
\begin{aligned}
\prod_{n \geq 1}\left(1-q^{n}\right)^{3}= & \sum_{n \geq 0}(-1)^{n}(2 n+1) q^{n(n+1) / 2} \\
= & 1-3 q+5 q^{3}-7 q^{6}+9 q^{10}-11 q^{15} \\
& \quad+13 q^{21}-15 q^{28}+17 q^{36}-19 q^{45}+\cdots .
\end{aligned}
$$

Modulo 5, this can be written as

$$
\begin{aligned}
\prod_{n \geq 1}\left(1-q^{n}\right)^{3} & \equiv\left(1-q^{10}-q^{15}+q^{45}+\cdots\right)-3 q\left(1-q^{5}-q^{20}+q^{35}+\cdots\right) \\
& \equiv A\left(q^{5}\right)-3 q B\left(q^{5}\right)
\end{aligned}
$$

for certain functions $A$ and $B$ of $q$. (It is a straightforward exercise to show that

$$
A(q)=\prod_{n \geq 1}\left(1-q^{5 n-3}\right)\left(1-q^{5 n-2}\right)\left(1-q^{5 n}\right)
$$

and

$$
B(q)=\prod_{n \geq 1}\left(1-q^{5 n-4}\right)\left(1-q^{5 n-1}\right)\left(1-q^{5 n}\right) .
$$

The interested reader may wish to see [6] for more information on how one proves these product representations of $A(q)$ and $B(q)$.) Thus, modulo 5,

$$
\prod_{n \geq 1}\left(1-q^{2 n}\right)^{3}\left(1-q^{n}\right)^{3} \equiv\left(A\left(q^{5}\right)-3 q B\left(q^{5}\right)\right)\left(A\left(q^{10}\right)-3 q^{2} B\left(q^{10}\right)\right) .
$$

From the above, we see that the coefficient of $q^{5 n+4}$ is divisible by 5 because the righthand side of the congruence contains no terms of the form $q^{5 n+4}$. Thanks to (2.8), we now know that, for all $n \geq 0$,

$$
h(10 n+9)=h(2(5 n+4)+1) \equiv 0(\bmod 5) .
$$

Lastly, for all $n \geq 1$, we know that $h(n)=f(3 n-2)$ thanks to (1.1) and (2.4). Therefore,

$$
f(30 n+25)=f(3(10 n+9)-2) \equiv 0(\bmod 5) .
$$

This completes the proof of Theorem 2.5.

\section{Closing thoughts}

Two sets of closing thoughts are in order. First, it would be instructive to obtain combinatorial proofs of results like those above based on the combinatorial 'structure' of the 1-shell TSPPs. Secondly, it appears that $f(n)$ satisfies congruences in arithmetic progression modulo 4 and 8 based on the computational evidence available. It would be desirable to see proofs of these results as well (whether combinatorial in flavour or via $q$-series manipulations). 


\section{Acknowledgement}

The authors gratefully acknowledge George Andrews for valuable comments during the completion of this work.

\section{References}

[1] G. E. Andrews, The Theory of Partitions (Addison-Wesley, Reading, MA, 1976) (reprinted Cambridge University Press, Cambridge, 1984, 1998).

[2] G. E. Andrews and B. C. Berndt, Ramanujan's Lost Notebook, Part I (Springer, New York, 2005).

[3] G. E. Andrews, P. Paule and C. Schneider, 'Plane partitions VI: Stembridge's TSPP theorem', Adv. Appl. Math. 34(4) (2005), 709-739.

[4] A. Blecher, 'Geometry for totally symmetric plane partitions (TSPPs) with self-conjugate main diagonal', Util. Math. 88 (2012), 223-235.

[5] N. J. Fine, Basic Hypergeometric Series and Applications, Mathematical Surveys and Monographs, 27 (American Mathematical Society, Providence, RI, 1988).

[6] M. D. Hirschhorn, 'Another short proof of Ramanujan's mod 5 partition congruence, and more', Amer. Math. Monthly 106(6) (1999), 580-583.

[7] J. R. Stembridge, 'The enumeration of totally symmetric plane partitions', Adv. Math. 111(2) (1995), 227-243.

\section{MICHAEL D. HIRSCHHORN, School of Mathematics and Statistics, University of New South Wales, Sydney 2052, Australia e-mail: m.hirschhorn@unsw.edu.au}

JAMES A. SELLERS, Department of Mathematics, Penn State University, University Park, PA 16802, USA

e-mail: sellersj@psu.edu 